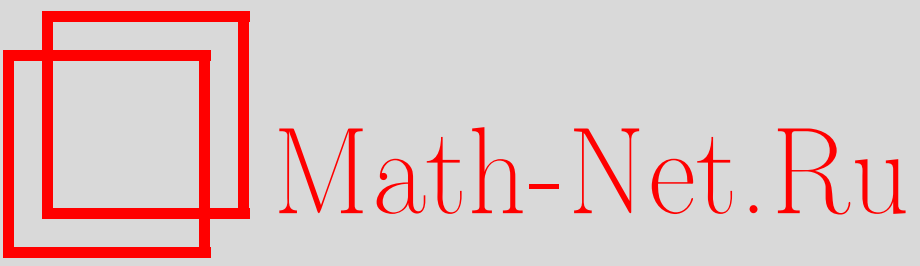

М. Ядипур, Ф. Хашемзаде, М. Барадараниа, Новый метод увеличения области притяжения аффинных нелинейных систем, Итоги науки и техн. Сер. Соврем. мат. и ее прил. Темат. обз., 2020, том 178, 91-101

DOI: https://doi.org/10.36535/0233-6723-2020-178-91-101

Использование Общероссийского математического портала Math-Net.Ru подразумевает, что вы прочитали и согласны с пользовательским соглашением

http: //www. mathnet.ru/rus/agreement

Параметры загрузки:

IP : 54.198 .67 .100

26 апреля 2023 г., 11:35:15 


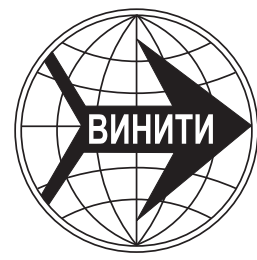

ИТОГИ НАУКИ И ТЕХНИКИ.

Современная математика и ее приложения.

Тематические обзоры.

Том 178 (2020). C. 91-101

DOI: $10.36535 / 0233-6723-2020-178-91-101$

УДК 517.977

\title{
НОВЫЙ МЕТОД УВЕЛИЧЕНИЯ ОБЛАСТИ ПРИТЯЖЕНИЯ АФФИННЫХ НЕЛИНЕЙНЫХ СИСТЕМ
}

\author{
(c) 2020 г. М. ЯДИПУР, Ф. ХАШЕМЗАДЕ, М. БАРАДАРАНИА
}

\begin{abstract}
АннотАция. Предлагается новый метод увеличения области притяжения для асимптотически устойчивой аффинной нелинейной системы. Метод основан на том, что образ такой нелинейной системы при непрерывном инъективном отображении асимптотически устойчив. Управление строится с помощью отображения области притяжения разомкнутой управляемой системы в бо́льшую область, являющуюся областью притяжения замкнутой управляемой системы. Получены требования к отображению, изменяющему форму и размер области притяжения нужным образом. Построение такого отображения проиллюстрировано на примерах. Эффективность предложенного метода подтверждена экспериментальными результатами.
\end{abstract}

Ключевъе слова: область притяжения, асимптотическая устойчивость, аффинная нелинейная система, инъективное отображение.

\section{A NOVEL STRATEGY TO ENLARGE THE DOMAIN OF ATTRACTION OF AFFINE NONLINEAR SYSTEMS}

\author{
(c) 2020 M. YADIPOUR, F. HASHEMZADEH, M. BARADARANNIA
}

\begin{abstract}
In this paper, a novel strategy to enlarge the domain of attraction of an asymptotically stable, affine nonlinear system is proposed. This method is based on the concept of mapping and relies on asymptotic stability of a nonlinear system image via a continuous and injective mapping. The goal of designing the controller is to map the domain of attraction of an open loop system into a larger domain, which is the domain of attraction of a closed loop system. To find a suitable map that can make desired changes in the shape and size of domain of attraction, a criterion is obtained and its implementation instruction is described through examples. Simulation results are provided to demonstrate the efficiency of the proposed method.
\end{abstract}

Keywords and phrases: domain of attraction, asymptotic stability, affine nonlinear system, injective mapping.

AMS Subject Classification: 93C15

1. Введение. Важнейшим вопросом анализа нелинейных динамических систем является вопрос устойчивости. Знания асимптотической устойчивости положения равновесия, как правило, недостаточно для приложений, так как необходимо учитывать, насколько далеко начальное состояние может быть от положения равновесия, чтобы сохранялась асимптотическая устойчивость. Это приводит к понятию области притяжения. Задача точного определения или оценки области притяжения стоит начиная с появляния теоремы об устойчивости в 1892 г. Вообще говоря, задача определения области притяжения (ОП) слишком сложна, поэтому также рассматривают задачу вычисления наибольшего инвариантного подмножества ОП. Методы оценки ОП разделяются на два класса: ляпуновские и неляпуновские методы (см. [14]). 
Точная ОП нелинейной системы может быть определена с помощью максимальной функции Ляпунова, а такая функция может быть приближена рациональной функцией Ляпунова (см. [8]). Квадратичные функции Ляпунова часто дают консервативные приближения области устойчивости. Тем не менее квадратичные функции Ляпунова используются в задачах стабилизации (см. [18]); также они используются для построения асимметричных функций Ляпунова для оценки ОП линейных систем относительно асимметричного внешнего воздействия (см. [15]). С другой стороны, исследования показывают, что произвольные функции Ляпунова, удовлетворяющие условиям Ляпунова, доставляют оценку ОП полиномиальных нелинейных управляемых систем (см. [23]). Класс подходящих для оценки ОП функций Ляпунова был расширен с помощью теоремы, доказанной в [9]. Был разработан новый подход к вычислению функций Ляпунова, имеющий больше степеней свободы по сравнению с классическим подходом (см. [7]). Чтобы использовать полиномиальные функции Ляпунова для оценки ОП неполиномиальных систем, необходимо приблизить неполиномиальную систему неточной полиномиальной. Некоторые методы приближения, основанные на полиномиальной многопараметрической технике анализа остаточной функции, были разработаны в $[12,28])$. Кусочно аффинные функции также могут быть использованы в качестве функций Ляпунова для оценки области притяжения (см. [19]).

Для исследования устойчивости нелинейных систем и построения новых оценок ОП широко применяются метод Зубова (см. [24]), критерий Пупова, круговой критерий, теорема ЛаСалля, теория центральных многообразий (см. [4]). В большинстве случаев задача оценки ОП сводится к задаче выпуклой или невыпуклой оптимизации (см. [2]) и решается с помощью таких оптимизационных методов, как LMI (см. $[5,6,22,29])$, SOS (см. [10,26]), интеллектуальных оптимизационных методов (см. [1]), комбинации LMI и генетического алгоритма (см. [11]). В противоположность оптимизационным методам существует быстрый и вычислительно эффективный подход, разработанный для приложений в реальном времени, при котором алгоритм ищет такое наибольшее подуровневое множество для функции Ляпунова, что ее производная по времени отрицательно определена (см. [20]).

Оценка области притяжения неточной системы может быть более сложной задачей, особенно для систем большой размерности (см. [30]). Внутренняя оценка ОП для класса неточных нелинейных систем может быть получена новым методом, основанным на параметрически независимых функциях Ляпунова (см. [27]), а также методом ветвей и границ (см. [25]). Более того, в [21] предложен новый подход к определению устойчивости скользящего режима управления в условиях неопределенности.

Для вычисления области притяжения используются меры заполнения, имеющие долгую историю применений в стохастическом управлении (см. $[13,17])$. Для оценки ОП также применяется неляпуновский метод обращения траекторий, основанный на топологических соображениях. Одним из его недостатков является вычислительная сложность, которую можно снизить, комбинируя этот метод с ляпуновскими методами (см. [16]). Другой неляпуновский подход к оценке области притяжения дает построение множеств достижимости. Приближенный вычислительный метод построения множеств достижимости приведен в [3].

В настоящей работе содержится новый подход к увеличению размера и изменению формы области притяжения для нелинейных систем. В разделе 2 предложен новый метод, основанный на рассмотрении непрерывных инъективных отображений, с помощью которых достигаются заявленные изменения области устойчивости. Кроме того, в том же разделе разработаны требования к подходящим отображениям. В разделе 3 доказано, что образ асимптотически устойчивой системы при непрерывном инъективном отображении также является асимптотически устойчивой системой. Раздел 4 содержит примеры, иллюстирующие требования к построению подходящего отображения и качество предложенного метода. В разделе 5 перечислены основные результаты работы.

2. Метод. В настоящем разделе предложен новый метод увеличения размера и изменения формы области притяжения нелинейной динамической системы. Для асимптотически устойчивой системы построен закон управления, переводящий область притяжения в целевую область. Тем 
самым задача увеличения или изменения формы ОП сводится к задаче нахождения подходящего отображения для специальной системы.

В настоящей работе рассматриваются нелинейные динамические системы следующего вида:

$$
\dot{\boldsymbol{x}}=f(\boldsymbol{x})+g(\boldsymbol{x}) u,
$$

где $f: \mathbb{R}^{n} \rightarrow \mathbb{R}^{n}$ и $g: \mathbb{R}^{n} \rightarrow \mathbb{R}^{n}$ - аналитические функции, $\boldsymbol{x} \in \mathbb{R}^{n}$ - переменная состояния, а $u: \mathbb{R}^{n} \rightarrow \mathbb{R}$ - управление системой.

Пусть $\boldsymbol{x}=0$ - асимптотически устойчивое положение равновесия системы $(2.1)$ при $u=0$. Цель состоит в построении управления для сохранения асимптотической устойчивости положения равновесия $\boldsymbol{x}=0$ и увеличения его области притяжения. Рассмотрим систему

$$
\dot{\boldsymbol{z}}=f(\boldsymbol{z}),
$$

где $f$-то же, что и в уравнении (2.1). Ясно, что $\boldsymbol{z}=0$ - асимптотически устойчивое положение равновесия системы (2.2). Пусть $M(\cdot)$ - непрерывное и инъективное отображение пространств $\boldsymbol{x}$ и $z$ :

$$
\boldsymbol{z}=M(\boldsymbol{x}) .
$$

Требуется выбрать непрерывное и инъективное отображение, переводящее ОП системы (2.2) в бо́льшую область в пространстве $\boldsymbol{x}$, а затем использовать управление $u(\cdot)$ системы $(2.1)$, чтобы перевести систему (2.1) в образ при отображении $M$ системы $(2.2)$ в пространстве $\boldsymbol{x}$. Имеется бесконечная серия отображений, увеличивающих площадь области притяжения, например, линейные растяжения с коэффициентом $<1$. Подходящее отображение должно удовлетворять двум требованиям. Во-первых, точка $\boldsymbol{x}=0$ должна оставаться положением равновесия замкнутой системы (2.1). Это означает, что точка $\boldsymbol{x}=0$ должна быть положением равновесия образа при отображении $M$ системы (2.2) в пространстве $\boldsymbol{x}$. Для достижения этого должно быть выполнено следующее условие:

$$
\left\{\begin{array}{l}
\boldsymbol{x}=0 \Leftrightarrow M(\boldsymbol{x})=0 \\
\operatorname{det}\left(\left.\frac{\delta M}{\delta \boldsymbol{x}}\right|_{\boldsymbol{x}=0}\right) \neq 0 .
\end{array}\right.
$$

Во-вторых, должно быть гарантировано существование управления ( $и$ в системе (2.1)), которое переводит систему (2.1) в образ системы (2.2) в пространстве $\boldsymbol{x}$. Соответствующее свойство для двумерных систем выводится ниже. Для систем больших размерностей процедура аналогична.

Запишем уравнения (2.1), (2.2) и (2.3) в двумерном случае следующим образом:

$$
\begin{aligned}
& \left\{\begin{array}{l}
\dot{x}_{1}=f_{1}(\boldsymbol{x})+g_{1}(\boldsymbol{x}) u, \\
\dot{x}_{2}=f_{2}(\boldsymbol{x})+g_{2}(\boldsymbol{x}) u ;
\end{array}\right. \\
& \left\{\begin{array}{l}
\dot{z}_{1}=f_{1}(\boldsymbol{z}), \\
\dot{z}_{2}=f_{2}(\boldsymbol{z}) ;
\end{array}\right. \\
& \left\{\begin{array}{l}
z_{1}=M_{1}(\boldsymbol{x}), \\
z_{2}=M_{2}(\boldsymbol{x}) .
\end{array}\right.
\end{aligned}
$$

Производная по времени уравнения (2.7) имеет вид

$$
\left\{\begin{array}{l}
\dot{z}_{1}=M_{1 x_{1}} \cdot \dot{x}_{1}+M_{1 x_{2}} \cdot \dot{x}_{2} \\
\dot{z}_{2}=M_{2 x_{1}} \cdot \dot{x}_{1}+M_{2 x_{2}} \cdot \dot{x}_{2}
\end{array}\right.
$$

где

$$
M_{i x_{1}}=\frac{\delta M_{i}}{\delta x_{1}}, \quad M_{i x_{2}}=\frac{\delta M_{i}}{\delta x_{2}}, \quad i=1,2 .
$$

Подставляя выражения (2.6) и (2.7) в уравнения (2.8), получим

$$
\left\{f_{1}(\boldsymbol{M})=M_{1 x_{1}} \cdot \dot{x}_{1}+M_{1 x_{2}} \cdot \dot{x}_{2}, \quad f_{2}(\boldsymbol{M})=M_{2 x_{1}} \cdot \dot{x}_{1}+M_{2 x_{2}} \cdot \dot{x}_{2}\right.
$$


Разрешая уравнения (2.9) относительно неизвестных $\dot{x}_{1}$ и $\dot{x}_{2}$, находим

$$
\begin{aligned}
& \dot{x}_{1}=\frac{M_{1 x_{2}} f_{2}(\boldsymbol{M})-M_{2 x_{2}} f_{1}(\boldsymbol{M})}{M_{1 x_{2}} M_{2 x_{1}}-M_{2 x_{2}} M_{1 x_{1}}}, \\
& \dot{x}_{2}=\frac{-M_{1 x_{1}} f_{2}(\boldsymbol{M})+M_{2 x_{1}} f_{1}(\boldsymbol{M})}{M_{1 x_{2}} M_{2 x_{1}}-M_{2 x_{2}} M_{1 x_{1}}} .
\end{aligned}
$$

Подставляя выражения (2.10) и (2.11) в уравнения (2.5), получим два выражения для управления $u$ :

$$
\begin{aligned}
& u=\frac{M_{1 x_{2}} f_{2}(\boldsymbol{M})-M_{2 x_{2}} f_{1}(\boldsymbol{M})-\left(M_{1 x_{2}} M_{2 x_{1}}-M_{2 x_{2}} M_{1 x_{1}}\right) f_{1}(\boldsymbol{x})}{g_{1}(\boldsymbol{x})\left(M_{1 x_{2}} M_{2 x_{1}}-M_{2 x_{2}} M_{1 x_{1}}\right)}, \\
& u=\frac{M_{1 x_{1}} f_{2}(\boldsymbol{M})-M_{2 x_{1}} f_{1}(\boldsymbol{M})-\left(M_{1 x_{1}} M_{2 x_{2}}-M_{2 x_{1}} M_{1 x_{2}}\right) f_{2}(\boldsymbol{x})}{g_{2}(\boldsymbol{x})\left(M_{1 x_{1}} M_{2 x_{2}}-M_{2 x_{1}} M_{1 x_{2}}\right)} .
\end{aligned}
$$

Ясно, что выражения (2.12) и (2.13) должны быть эквивалентны. Это доставляет второе свойство, выраженное в терминах величин $g_{i}, i=1,2$ :

$$
\begin{gathered}
\frac{g_{1}}{g_{2}}=-\frac{M_{1 x_{2}} f_{2}(\boldsymbol{M})-M_{2 x_{2}} f_{1}(\boldsymbol{M})-\left(M_{1 x_{2}} M_{2 x_{1}}-M_{2 x_{2}} M_{1 x_{1}}\right) f_{1}(\boldsymbol{x})}{M_{1 x_{1}} f_{2}(\boldsymbol{M})-M_{2 x_{1}} f_{1}(\boldsymbol{M})-\left(M_{1 x_{1}} M_{2 x_{2}}-M_{2 x_{1}} M_{1 x_{2}}\right) f_{2}(\boldsymbol{x})}, \quad g_{1} \neq 0, g_{2} \neq 0, \\
M_{1 x_{2}} f_{2}(\boldsymbol{M})-M_{2 x_{2}} f_{1}(\boldsymbol{M})-\left(M_{1 x_{2}} M_{2 x_{1}}-M_{2 x_{2}} M_{1 x_{1}}\right) f_{1}(\boldsymbol{x})=0, \quad g_{1}=0, \\
M_{1 x_{1}} f_{2}(\boldsymbol{M})-M_{2 x_{1}} f_{1}(\boldsymbol{M})-\left(M_{1 x_{1}} M_{2 x_{2}}-M_{2 x_{1}} M_{1 x_{2}}\right) f_{2}(\boldsymbol{x})=0, \quad g_{2}=0 .
\end{gathered}
$$

Таким образом, наше отображение должно удовлетворять требованию (2.4) и одному из (2.14), (2.15) или (2.16). Следовательно, разумный подход к уравнению (2.14) заключается в выборе $M_{1}(\boldsymbol{x})$ и подстановке его в уравнение $(2.14)$ для получения $M_{2}(\boldsymbol{x})$ и наоборот. Ясно, что $M_{1}(\boldsymbol{x})$ соответствует перемещению точек ОП в направлении $x_{1}$, а $M_{2}(\boldsymbol{x})$ управляет перемещением точек в направлении $x_{2}$. Например, использование $M_{1}(\boldsymbol{x})=k_{1} x_{1}, 0<k_{1}<1$, расширяет область устойчивости вдоль оси $x$. Подставляя это выражение в уравнение $(2.14)$, получим семейство подходящих отображений, одно из которых можно выбрать исходя из предъявляемых требований. Более того, в предыдущем примере, если $M_{2}(\boldsymbol{x})=k_{2} x_{2}, 0<k_{2}<1$, подходит, то отображение

$$
M(\boldsymbol{x})=\left(\begin{array}{cc}
k_{1} & 0 \\
0 & k_{2}
\end{array}\right) \boldsymbol{x}, \quad k_{1}, k_{2} \in \mathbb{R}^{+}, \quad k_{1}, k_{2}<1,
$$

может быть использовано для увеличения области устойчивости в обоих направлениях. Между тем выбор $k_{1}=k_{2}$ сохраняет форму области устойчивости и увеличивает ее размер.

В случае, если отображение (2.17) не удовлетворяет требованию (2.14), управление системой (2.1) может быть модифицировано так, чтобы отображение (2.17) было применимо к системе (2.1). Рассмотрим управление системой (2.1) вида

$$
u(\boldsymbol{x})=v_{1}(\boldsymbol{x})+v_{2}(\boldsymbol{x}) .
$$

Цель заключается в построении управления $v_{1}$, при котором замкнутая система (2.1) удовлетворяет требованию (2.14) при отображении (2.17). После достижения этой цели, управление $v_{2}$ может быть вычислено и использовано для отображения области устойчивости замкнутой управляемой системы в новую область, определенную с помощью отображения (2.17). Подставляя выражение $(2.18)$ в уравнения $(2.5)$, получим

$$
\left\{\begin{array}{l}
\dot{x}_{1}=\left(f_{1}(\boldsymbol{x})+g_{1}(\boldsymbol{x}) v_{1}(\boldsymbol{x})\right)+g_{1}(\boldsymbol{x}) v_{2}(\boldsymbol{x}), \\
\dot{x}_{2}=\left(f_{2}(\boldsymbol{x})+g_{2}(\boldsymbol{x}) v_{1}(\boldsymbol{x})\right)+g_{2}(\boldsymbol{x}) v_{2}(\boldsymbol{x}),
\end{array}\right.
$$

а также систему (2.6) в виде

$$
\left\{\begin{array}{l}
\dot{z}_{1}=f_{1}(\boldsymbol{z})+g_{1}(\boldsymbol{z}) v_{1}(\boldsymbol{z}) \\
\dot{z}_{2}=f_{2}(\boldsymbol{z})+g_{2}(\boldsymbol{z}) v_{1}(\boldsymbol{z})
\end{array}\right.
$$


Перепишем уравнения (2.17), (2.8) в виде

$$
\left\{\begin{array}{l}
\dot{z}_{1}=k_{1} \dot{x}_{1} \\
\dot{z}_{2}=k_{2} \dot{x}_{2}
\end{array}\right.
$$

а уравнения $(2.20)$ - в виде

$$
\left\{\begin{array}{l}
\dot{z}_{1}=f_{1}(\boldsymbol{M})+g_{1}(\boldsymbol{M}) v_{1}(\boldsymbol{M}), \\
\dot{z}_{2}=f_{2}(\boldsymbol{M})+g_{2}(\boldsymbol{M}) v_{1}(\boldsymbol{M}) .
\end{array}\right.
$$

Подставляя выражения (2.19) и (2.22) в уравнения (2.21), получим два выражения для $v_{2}(\boldsymbol{x})$ следующего вида:

$$
\left\{\begin{array}{l}
v_{2}(\boldsymbol{x})=\frac{f_{1}(\boldsymbol{M})}{k_{1} g_{1}(\boldsymbol{x})}+\frac{g_{1}(\boldsymbol{M}) v_{1}(\boldsymbol{M})}{k_{1} g_{1}(\boldsymbol{x})}-\frac{f_{1}(\boldsymbol{x})}{g_{1}(\boldsymbol{x})}-v_{1}(\boldsymbol{x}), \\
v_{2}(\boldsymbol{x})=\frac{f_{2}(\boldsymbol{M})}{k_{2} g_{2}(\boldsymbol{x})}+\frac{g_{2}(\boldsymbol{M}) v_{1}(\boldsymbol{M})}{k_{2} g_{2}(\boldsymbol{x})}-\frac{f_{2}(\boldsymbol{x})}{g_{2}(\boldsymbol{x})}-v_{1}(\boldsymbol{x}) .
\end{array}\right.
$$

Приравнивая выражения (2.23), получим выражение для $v_{1}(\boldsymbol{x})$ вида

$$
v_{1}(\boldsymbol{x})=\frac{\frac{f_{2}(\boldsymbol{x})}{k_{2} g_{2}\left(\frac{x_{1}}{k_{1}}, \frac{x_{2}}{k_{2}}\right)}-\frac{f_{2}\left(\frac{x_{1}}{k_{1}}, \frac{x_{2}}{k_{2}}\right)}{g_{2}\left(\frac{x_{1}}{k_{1}}, \frac{x_{2}}{k_{2}}\right)}-\frac{f_{1}(\boldsymbol{x})}{k_{1} g_{1}\left(\frac{x_{1}}{k_{1}}, \frac{x_{2}}{k_{2}}\right)}+\frac{f_{1}\left(\frac{x_{1}}{k_{1}}, \frac{x_{2}}{k_{2}}\right)}{g_{1}\left(\frac{x_{1}}{k_{1}}, \frac{x_{2}}{k_{2}}\right)}}{\frac{g_{1}(\boldsymbol{x})}{k_{1} g_{1}\left(\frac{x_{1}}{k_{1}}, \frac{x_{2}}{k_{2}}\right)}-\frac{g_{2}(\boldsymbol{x})}{k_{2} g_{2}\left(\frac{x_{1}}{k_{1}}, \frac{x_{2}}{k_{2}}\right)}} .
$$

Подставляя выражение (2.24), например, в первое из уравнений (2.23), получим

$$
v_{2}(\boldsymbol{x})=\frac{f_{1}\left(k_{1} x_{1}, k_{2} x_{2}\right)+g_{1}\left(k_{1} x_{1}, k_{2} x_{2}\right) v_{1}\left(k_{1} x_{1}, k_{2} x_{2}\right)}{k_{1} g_{1}(\boldsymbol{x})}-\frac{f_{1}(\boldsymbol{x})}{g_{1}(\boldsymbol{x})}-v_{1}(\boldsymbol{x}) .
$$

3. Анализ устойчивости. Реализация описанного подхода зависит от асимптотической устойчивости замкнутой управляемой системы (2.1). Используя предложенное управление (2.12) или (2.13), легко видеть, что замкнутая система (2.1) есть образ системы (2.2) в пространстве $\boldsymbol{x}$ относительно отображения $M$. Таким образом, достаточно доказать асимптотическую устойчивость системы-образа. Более точно, нужно показать, что если нелинейная система асимптотически устойчива, то ее образ относительно непрерывного инъективного отображения также асимптотически устойчив. Это утверждение, выглядящее интуитивно очевидным, сформулировано в виде теоремы и доказано ниже.

Теорема 1. Пусть система $\dot{\boldsymbol{z}}=f(\boldsymbol{z})$ асимптотически устойчива в окрестности положения равновесия $\boldsymbol{z}=0$ и $\dot{\boldsymbol{x}}=f(\boldsymbol{x})$ есть ее образ относительно такого непрерывного инбективного отображения $\boldsymbol{z}=M(\boldsymbol{x})$, что

$$
M(0)=0, \quad \operatorname{det}\left(\left.\frac{\delta M}{\delta \boldsymbol{x}}\right|_{\boldsymbol{x}=0}\right) \neq 0 .
$$

Тогда точка $\boldsymbol{x}=0$-асимптотически устойчивое положение равновесия системы-образа $\dot{\boldsymbol{x}}=$ $f(\boldsymbol{x})$.

Доказательство. Вычисляя производную по времени от $\boldsymbol{z}=M(\boldsymbol{x})$, получим

$$
\frac{\delta M}{\delta \boldsymbol{x}} \dot{\boldsymbol{x}}=f b(M(\boldsymbol{x})) .
$$

Так как $\boldsymbol{z}=0$ есть положение равновесия системы $\dot{\boldsymbol{z}}=f(\boldsymbol{z})$, то $f(M(0))=0$. Далее, согласно предположению (2.4),

$$
\operatorname{det}\left(\left.\frac{\delta M}{\delta \boldsymbol{x}}\right|_{\boldsymbol{x}=0}\right) \neq\left. 0 \Rightarrow \frac{\delta M}{\delta \boldsymbol{x}}\right|_{\boldsymbol{x}=0} \neq 0
$$

легко видеть, что если $\boldsymbol{x}=0$, то $\dot{\boldsymbol{x}}=0$. Значит, точка $\boldsymbol{x}=0$ есть положение равновесия системы $\dot{\boldsymbol{x}}=f(\boldsymbol{x})$. 
Используя предположение об устойчивости системы $\dot{\boldsymbol{z}}=f(\boldsymbol{z})$, можно заключить, что

$$
\forall \varepsilon_{z}>0 \exists \delta_{z}>0:\|z(0)\|<\delta_{z} \Rightarrow\|z(t)\|<\varepsilon_{z} \forall t>0 .
$$

Так как отображение $M(\cdot)$ непрерывно и инъективно, то образ относительно отображения $M$ связной и ограниченной области также связен и ограничен. Значит, для любого $\varepsilon_{x}>0$ существует по крайней мере одно такое $\varepsilon_{z}>0$, что

$$
\max _{\|\boldsymbol{z}\|<\varepsilon_{z}}\|\boldsymbol{x}\|=\varepsilon_{x}
$$

кроме того, ясно, что $\min _{\|\boldsymbol{z}\|<\varepsilon_{z}}\|\boldsymbol{x}\|=0$.

Из непрерывности и инъективности отображения $M$ следует, что обратное отображение $M^{-1}(\boldsymbol{z})$ также непрерывно и инъективно. Аналогично, для любого $\delta_{z}>0$ найдется по крайней мере одно $\delta_{x}>0$, для которого

$$
\max _{\|\boldsymbol{x}\|<\delta_{x}}\|\boldsymbol{z}\|=\delta_{z}
$$

Далее, для любого $\varepsilon_{x}>0$ существует $\varepsilon_{z}>0$; на основании устойчивости системы $\dot{\boldsymbol{z}}=f(\boldsymbol{z})$ существует $\delta_{z}>0$; соответственно, существует такое $\delta_{x}>0$, что выполнены (3.2), (3.3) и (3.4). Следовательно,

$$
\|x(0)\|<\delta_{x} \quad \Rightarrow \quad\|z(0)\|<\delta_{z} \quad \Rightarrow \quad\|z(t)\|<\varepsilon_{z} \quad \Rightarrow \quad\|x(t)\|<\varepsilon_{x},
$$

откуда можно заключить, что

$$
\|x(0)\|<\delta_{x} \quad \Rightarrow \quad\|x(t)\|<\varepsilon_{x} \quad \forall t>0 .
$$

Это доказывает устойчивость системы-образа $\dot{\boldsymbol{x}}=f(\boldsymbol{x})$.

Асимптотическую устойчивость системы-образа $\dot{\boldsymbol{x}}=f(\boldsymbol{x})$ можно легко вывести из асимптотической устойчивости системы $\dot{\boldsymbol{z}}=f(\boldsymbol{z})$. Предположим, что существует такое $\delta_{z}>0$, что

$$
\|z(0)\|<\delta_{z} \Rightarrow \lim _{t \rightarrow \infty} z(t)=0 .
$$

Так как образ окрестности вида $\|\boldsymbol{z}\|<\delta_{z}$ в пространстве $\boldsymbol{x}$ относительно непрерывного инъективного отображения $\boldsymbol{x}=M^{-1}(\boldsymbol{z})$ связен, то для любой окрестности вида $\|\boldsymbol{x}\|<\delta_{x}$, которая является подмножеством образа $\|\boldsymbol{z}\|<\delta_{z}$ в пространстве $\boldsymbol{x}$, используя (3.7), имеем

$$
\lim _{t \rightarrow \infty} x(t)=\lim _{t \rightarrow \infty} M^{-1}(z(t))=M^{-1}\left(\lim _{t \rightarrow \infty} z(t)\right)=M^{-1}(0)=0 \Rightarrow \lim _{t \rightarrow \infty} x(t)=0 .
$$

Значит система-образ $\dot{\boldsymbol{x}}=f(\boldsymbol{x})$ асимптотически устойчива.

4. Результаты экспериментов. В настоящем разделе рассмотрим три примера использования предложенного метода увеличения области притяжения нелинейных систем.

Пример 1. Рассмотрим обратную систему Ван дер Поля

$$
\left\{\begin{array}{l}
\dot{x}_{1}=x_{2}, \\
\dot{x}_{2}=-x_{1}-0,2 x_{2}+0,2 x_{1}^{2} x_{2}+u .
\end{array} .\right.
$$

Требуется найти отображение, удовлетворяющее требованиям (2.4) и (2.14), увеличивающее область притяжения системы (4.1). Будем искать отображение в виде

$$
\boldsymbol{z}=\boldsymbol{M}(\boldsymbol{x}), \quad z_{1}(\boldsymbol{x})=p x_{1}, z_{2}(\boldsymbol{x})=\lambda\left(x_{2}\right), \quad p \in \mathbb{R}, 0<p<1,
$$

где $z_{2}(\boldsymbol{x})=\lambda\left(x_{2}\right)$ - неизвестная компонента отображения, определяемая с помощью уравнения (2.15). Подставляя выражение (4.2) в уравнение (2.15), получим

$$
\lambda\left(x_{2}\right)=p x_{2}, \quad \boldsymbol{M}(\boldsymbol{x})=p \boldsymbol{x} .
$$

При увеличении модуля коэффициента это отображение увеличивает размер ОП, сохраняя его форму. Применяя построенное отображение к уравнению (2.13), находим управление

$$
u=0,2\left(p^{2}-1\right) x_{1}^{2} x_{2} .
$$




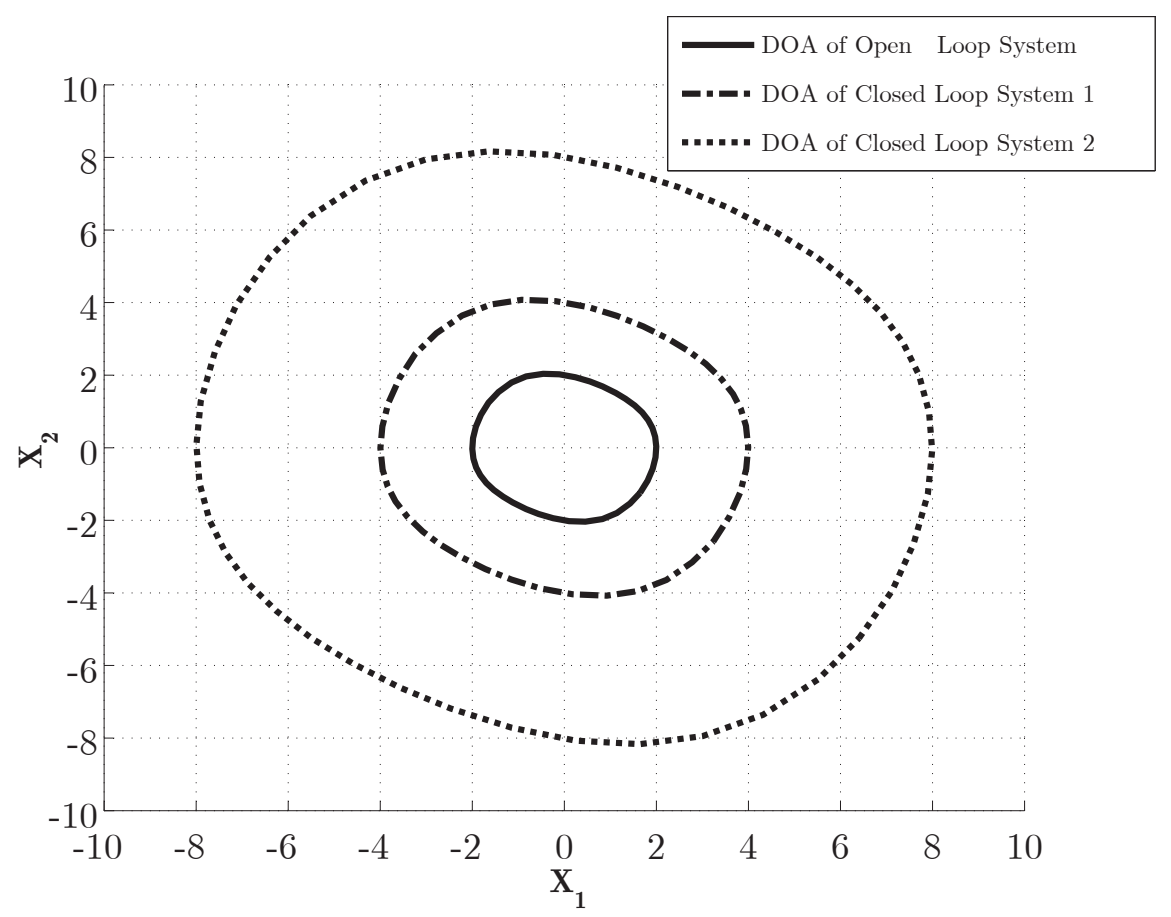

Рис. 1. Область притяжения системы (4.1) для $u=0$ в случае управления (4.4) при $p=0,5$ (замкнутая управляемая система 1), управления (4.4) при $p=0,25$ (замкнутая управляемая система 2).

Области притяжения системы (4.1) для $u=0$ и для управления (4.4) при $p=0,5$ и $p=$ 0,25 показаны на рис. 1 . Эти результаты согласуются с теоретическими ожиданиями, поскольку демонстрируют рост размера ОП при сохранении ее формы.

Пример 2. Рассмотрим двумерную систему (см. [5])

$$
\left\{\begin{array}{l}
\dot{x}_{1}=-x_{1}+x_{2}-x_{1}^{2}-x_{2}^{3}+x_{1} u, \\
\dot{x}_{2}=-2 x_{2}-x_{1}^{2}+u
\end{array}\right.
$$

выберем отображение вида (2.17). Используя формулы (2.18)-(2.25), получим

$$
\begin{aligned}
& v_{1}(\boldsymbol{x})=\frac{k_{2}}{k_{2}-1}\left(\frac{k_{2}-k_{1}^{2}}{k_{2} k_{1}^{2}} x_{1}^{2}+\frac{k_{1}-k_{2}}{k_{2}} \frac{x_{2}}{x_{1}}+\frac{k_{1}-1}{k_{1}} x_{1}+\frac{k_{2}^{3}-k_{1}}{k_{2}^{3}} \frac{x_{2}^{3}}{x_{1}}\right), \\
& v_{2}(\boldsymbol{x})=-v_{1}(\boldsymbol{x})+\frac{k_{2}-k_{1}^{2}}{k_{2}-1} x_{1}^{2}+\frac{k_{1}-k_{2}}{k_{1}\left(k_{2}-1\right)} \frac{x_{2}}{x_{1}}+\frac{k_{1}-1}{k_{2}-1} x_{1}+\frac{k_{2}^{3}-k_{1}}{k_{1}\left(k_{2}-1\right)} \frac{x_{2}^{3}}{x_{1}} .
\end{aligned}
$$

Так как выражения (4.6) и (??) имеют особенности в точке $x_{1}=0$, то управление системой (4.5) может быть выбрано так, что

$$
u(\boldsymbol{x})= \begin{cases}v_{1}(\boldsymbol{x})+v_{2}(\boldsymbol{x}), & \left|x_{1}\right| \geqslant \varepsilon \\ 0, & \left|x_{1}\right|<\varepsilon\end{cases}
$$

что увеличивает область притяжения системы (4.5) полуглобально. Области притяжения системы (4.5) для $u=0$ и для замкнутой управляемой системы (4.7) при $k_{1}=k_{2}=0,5$ и $\varepsilon=10^{-6}$ показаны на рис. 2. 


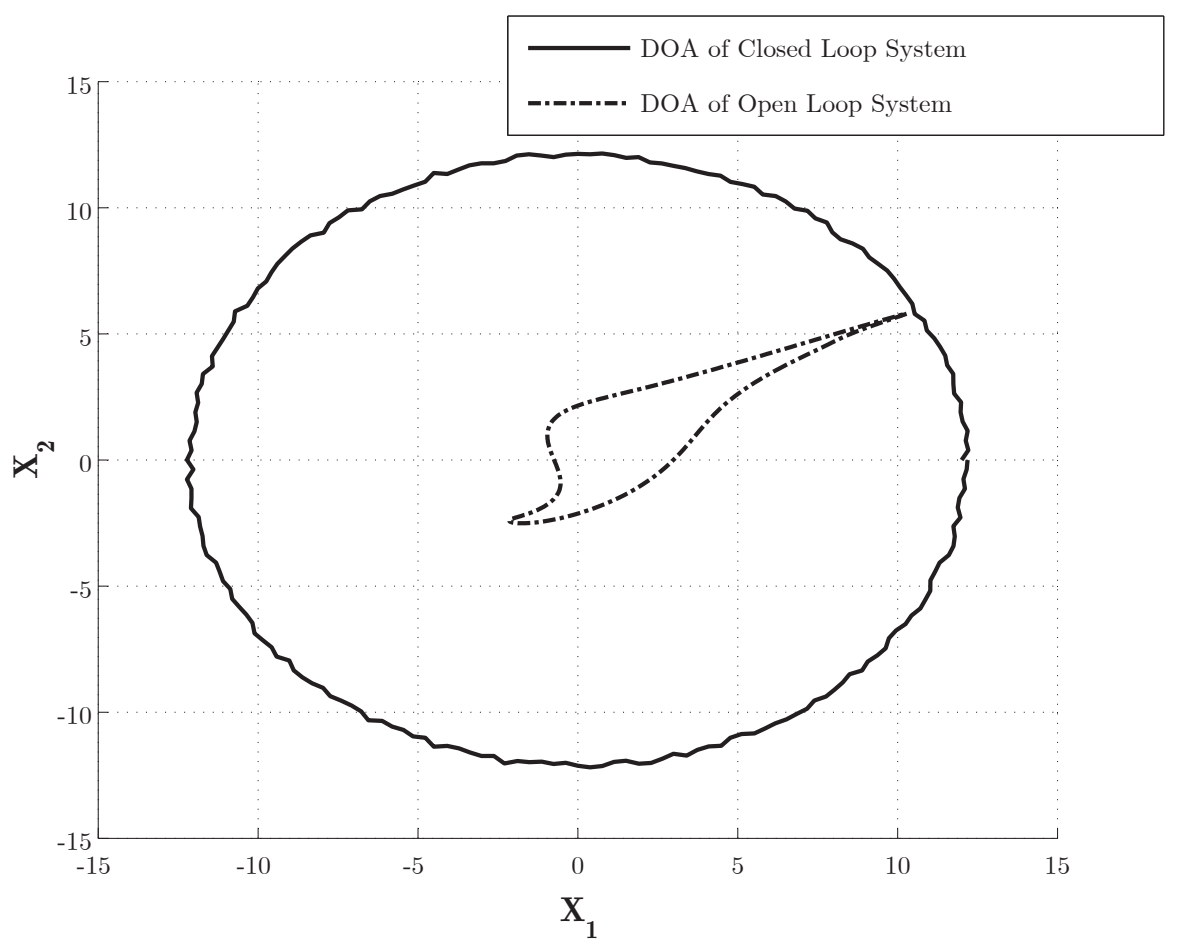

Рис. 2. Область притяжения системы (4.5) для $u=0$ и для управления (4.7) при $k_{1}=k_{2}=0,5$ $\varepsilon=10^{-6}$ (замкнутая управляемая система).

Пример 3. Предложенный метод может быть применим и к многомерным системам. Рассмотрим трехмерную нелинейную систему

$$
\left\{\begin{array}{l}
\dot{x}_{1}=-x_{1}+x_{2}^{3}+\frac{2+x_{3}^{2}}{1+x_{3}^{2}} u \\
\dot{x}_{2}=-x_{2}+x_{3} \\
\dot{x}_{3}=x_{1} x_{3}-x_{3}+u
\end{array}\right.
$$

Для этой системы отображение (2.17) может быть записано в виде

$$
M(\boldsymbol{x})=\left(\begin{array}{ccc}
k_{1} & 0 & 0 \\
0 & k_{2} & 0 \\
0 & 0 & k_{3}
\end{array}\right) \boldsymbol{x}, \quad k_{1}, k_{2}, k_{3} \in \mathbb{R}^{+}, \quad k_{1}, k_{2}, k_{3}<1 .
$$

Представим управление системой (4.8) в виде

$$
u(\boldsymbol{x})=v_{1}(\boldsymbol{x})+v_{2}(\boldsymbol{x}),
$$

получаем

$$
\left\{\begin{array}{l}
\dot{x}_{1}=\left(-x_{1}+x_{2}^{3}+\frac{2+x_{3}^{2}}{1+x_{3}^{2}} v_{1}(\boldsymbol{x})\right)+\frac{2+x_{3}^{2}}{1+x_{3}^{2}} v_{2}(\boldsymbol{x}), \\
\dot{x}_{2}=-x_{2}+x_{3} \\
\dot{x}_{3}=\left(x_{1} x_{3}-x_{3}+v_{1}(\boldsymbol{x})\right)+v_{2}(\boldsymbol{x}) .
\end{array}\right.
$$

Перепишем уравнения $(2.8)$ и $(2.9)$ в виде

$$
\left\{\begin{array} { l } 
{ \dot { z } _ { 1 } = k _ { 1 } \dot { x } _ { 1 } , } \\
{ \dot { z } _ { 2 } = k _ { 2 } \dot { x } _ { 2 } , } \\
{ \dot { z } _ { 3 } = k _ { 3 } \dot { x } _ { 3 } , }
\end{array} \quad \left\{\begin{array}{l}
\dot{z}_{1}=f_{1}(\boldsymbol{M})+g_{1}(\boldsymbol{M}) v_{1}(\boldsymbol{M}), \\
\dot{z}_{2}=f_{2}(\boldsymbol{M}), \\
\dot{z}_{3}=f_{3}(\boldsymbol{M})+g_{3}(\boldsymbol{M}) v_{1}(\boldsymbol{M}) .
\end{array}\right.\right.
$$




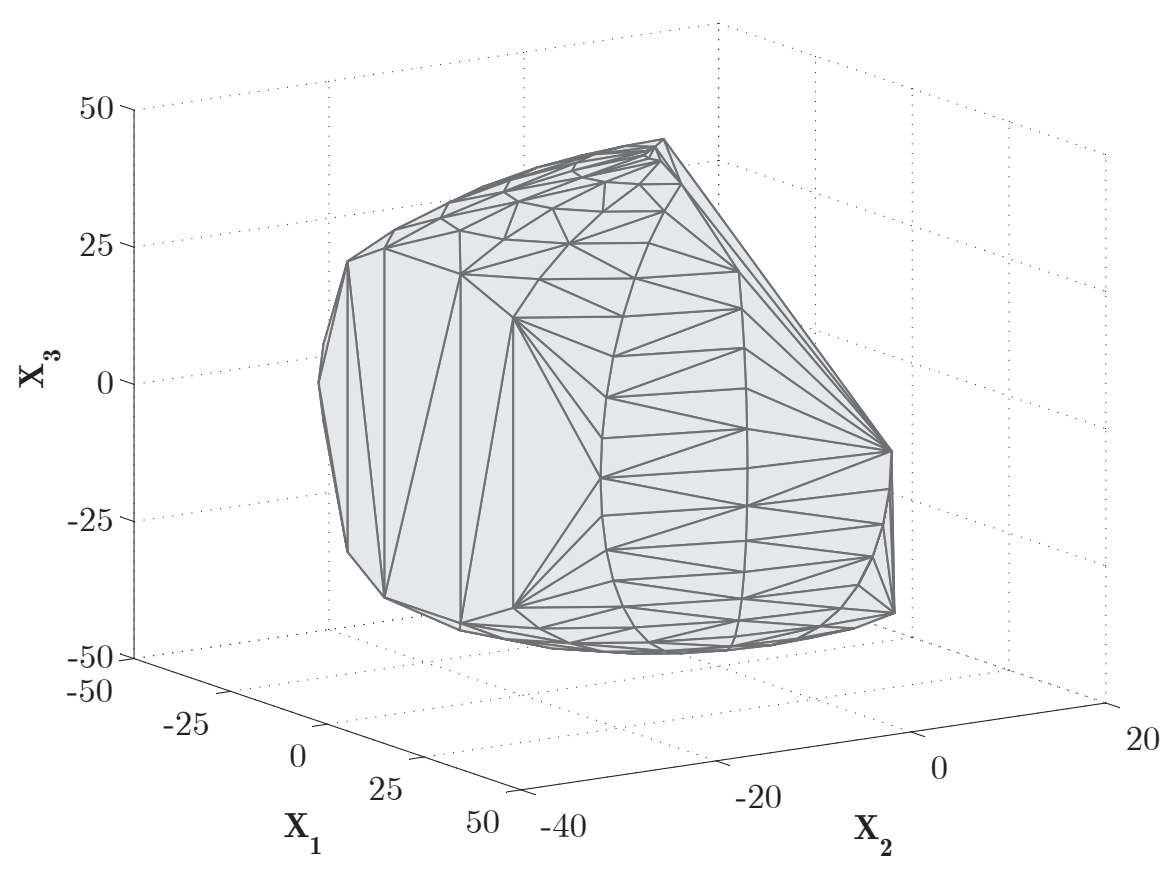

Рис. 3. Область притяжения системы (4.8) для $u=0$.

Следуя описанной процедуре, из уравнений (2.23) получим $k_{2}=k_{3}$ и

$$
\begin{aligned}
& v_{1}(\boldsymbol{x})=\frac{\frac{k_{1}-1}{k_{1} k_{2}} x_{1} x_{3}+k_{2}^{2} x_{2}^{3}\left(\frac{1}{k_{2}^{3}}-\frac{1}{k_{1}}\right)\left(1+\frac{x_{3}^{2}}{k_{2}^{2}}\right) /\left(2 k_{2}^{2}+x_{3}^{2}\right)}{\frac{\left(2+x_{3}^{2}\right)\left(1+\left(x_{3} / k_{2}\right)^{2}\right)}{k_{1}\left(1+x_{3}^{2}\right)\left(2+\left(x_{3} / k_{2}\right)^{2}\right)}-\frac{1}{k_{2}}}, \\
& v_{2}(\boldsymbol{x})=-v_{1}(\boldsymbol{x})+\frac{\left(k_{2}^{3}-k_{1}\right)\left(1+x_{3}^{2}\right) x_{2}^{3}}{2 k_{1}+k_{1} x_{3}^{2}}+\frac{\left(k_{1}-1\right) x_{1} x_{3}+\frac{\left(k_{1}-k_{2}^{3}\right)\left(1+x_{3}^{2}\right) x_{2}^{3}}{2 k_{1}+k_{1} x_{3}^{2}}}{1-\frac{k_{1}\left(1+k_{2}^{2} x_{3}^{2}\right)\left(2+x_{3}^{2}\right)}{k_{2}\left(2+k_{2}^{2} x_{3}^{2}\right)\left(1+x_{3}^{2}\right)}} .
\end{aligned}
$$

Области притяжения системы (4.8) для $u=0$ и для управления (4.10) при $k_{1}=0,8, k_{2}=0,09$ показаны на рис. 3 и 4, соответственно. Легко видеть, что при использовании управления область устойчивости может быть расширена.

5. Заключение. В настоящей работе предложен новый способ увеличения размера и изменения формы области притяжения нелинейных систем. Подход основан на построении непрерывного инъективного отображения. Доказано, что образ асимптотически устойчивой системы при непрерывном инъективном отображении является асимптотически устойчивой системой. Выведены требования к управлению, отображающему область притяжения асимптотически устойчивой нелинейной системы в бо́льшую область притяжения замкнутой управляемой системы. Таким образом, задача заключается в выборе подходящего отображения. Построение отображения, удовлетворяющего этим требованиям, требует решения сложного дифференциального уравнения в частных производных. Чтобы избежать этих сложностей, исходный подход модифицирован, чтобы строить простые линейные отображения с постоянными коэффициентами для увеличения области притяжения полуглобально.

Базовый подход проиллюстрирован на примере системы Ван дер Поля. Два других разобранных примера служат для иллюстрации работы модифицированного метода. Экспериментальные 


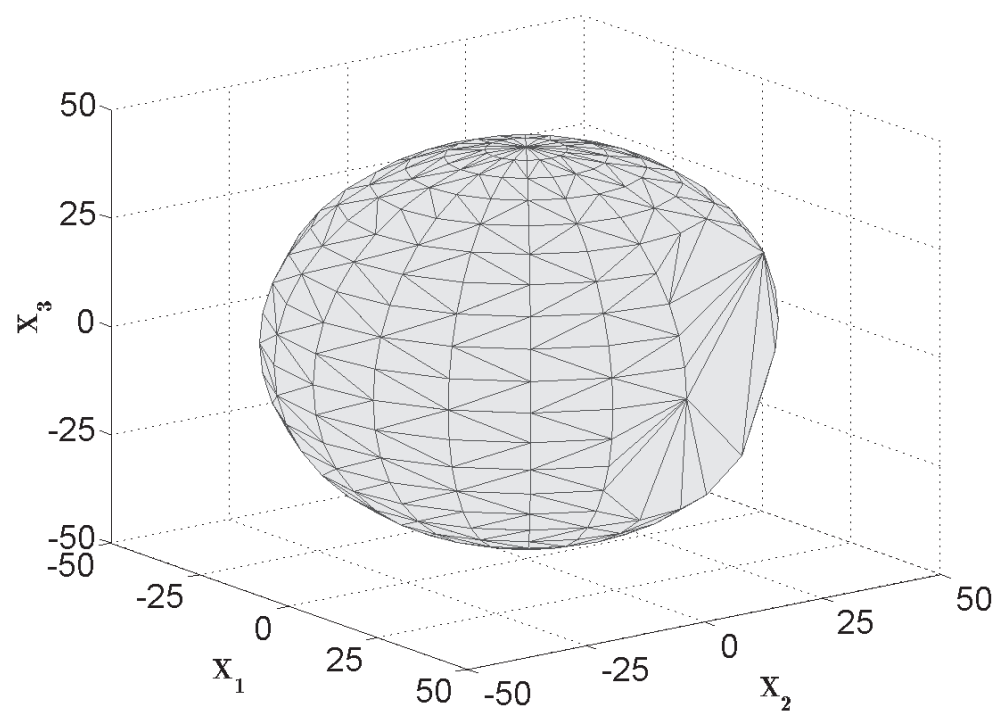

Рис. 4. Область притяжения системы (4.8) для управления (4.10) при $k_{1}=0,8, k_{2}=0,09$.

результаты соответствуют теоретическим ожиданиям и демонстрируют эффективность предложенного метода.

\section{СПИСОК ЛИТЕРАТУРЫ}

1. Amte A. Y., Kate P. S. Automatic generation of Lyapunov function using genetic programming approach// in: Proc. 2015 Int. Conf. on Energy Systems and Applications. - Pune, India 3: Dr. D. Y. Patil Inst. Eng. Technol., 2015. - P. 771-775.

2. Athanasopoulos N., Jungers R. M. Computing the domain of attraction of switching systems subject to non-convex constraints// in: Proc. 19th Int. Conf. on Hybrid Systems: Computation and Control. - New York: Association for Computing Machinery, 2016. - P. 41-50.

3. Baier R., Gerdts M. A computational method for non-convex reachable sets using optimal control// in: Proc. Eur. Control Conference 2009. - Budapest, 2009. - P. 97-102.

4. Cavoretto R., De Rossi A., Perracchione E., Venturino E. Robust approximation algorithms for the detection of attraction basins in dynamical systems// J. Sci. Comput. - 2016. - 68. - P. 395-415.

5. Chesi $G$. Computing output feedback controllers to enlarge the domain of attraction in polynomial systems// IEEE Trans. Automat. Control. - 2004. - 49. - P. 1846-1853.

6. Chesi G. Estimating the domain of attraction for non-polynomial systems via LMI optimizations// Automatica. - 2009. - 45. - P. 1536-1541.

7. Doban A. I., Lazar M. Computation of Lyapunov functions for nonlinear differential equations via a Masseratype construction 1603.03287, 2016./ arXiv: arXiv:1603.03287 [math.DS] .

8. Doban A. I., Lazar M. Feedback stabilization via rational control Lyapunov functions// in: Proc. 54th IEEE Conf. on Decision and Control. - Osaka, Japan, 2015. - P. 1148-1153.

9. Ermolin V. S., Vlasova T. V. Identification of the domain of attraction// in: Proc. Int. Conf. "Stability and Control Processes. - IEEE, 2015. - P. 9-12.

10. Franze G., Famularo D., Casavola A. Constrained nonlinear polynomial time-delay systems: A sum-ofsquares approach to estimate the domain of attraction// IEEE Trans. Automat. Control. - 2012. - 57. P. 2673-2679.

11. Hamidi F., Jerbi H., Aggoune W., Djemai M., Abdelkrim M. N. Enlarging the domain of attraction in nonlinear polynomial systems// Int. J. Comput. Commun. Control. - 2013. - 8. - P. 538-547.

12. Han D., Althoff $M$. Control synthesis for non-polynomial systems: A domain of attraction perspective// in: Proc. 54th IEEE Conf. on Decision and Control. - Osaka, Japan, 2015. - P. 1160-1167.

13. Henrion D., Korda M. Convex computation of the region of attraction of polynomial control systems// IEEE Trans. Automat. Control. - 2014. - 59. - P. 297-312. 
14. Khalil H. K. Noninear Systems. - Upper Saddle River, New Jersey: Prentice-Hall, 1996.

15. Li Y., Lin Z. On the estimation of the domain of attraction for linear systems with asymmetric actuator saturation via asymmetric Lyapunov functions// in: Proc. 2016 Am. Control Conf.. — Boston, 2016. P. 1136-1141.

16. Loccufier M., Noldus E. A new trajectory reversing method for estimating stability regions of autonomous nonlinear systems// Nonlin. Dynam. - 2000. - 21. - P. 265-288.

17. Majumdar A., Vasudevan R., Tobenkin M. M., Tedrake R. Convex optimization of nonlinear feedback controllers via occupation measures// Int. J. Robotics Res. - 2014. - 33. - P. 1209-1230.

18. Matallana L.G., Blanco A.M., Bandoni J. A. Nonlinear dynamic systems design based on the optimization of the domain of attraction// Math. Comput. Model. - 2011. - 53. - P. 731-745.

19. Milani B. E. Piecewise-affine Lyapunov functions for discrete-time linear systems with saturating controls// Automatica. - 2002. - 38. - P. 2177-2184.

20. Najafi E., Babuska R., Lopes G. A. A fast sampling method for estimating the domain of attraction// Nonlin. Dynam. - 2016. - 86. - P. 823-834.

21. Nersesov S.G., Ashrafiuon H., Ghorbanian P. On estimation of the domain of attraction for sliding mode control of underactuated nonlinear systems// Int. J. Robust Nonlin. Control. - 2014. - 24. - P. 811-824.

22. Polcz P., Szederkenyi G., Peni T. An improved method for estimating the domain of attraction of nonlinear systems containing rational functions// J. Phys. Conf. Ser. - 2015. - 659. - 012038.

23. Pursche T., Swiatlak R., Tibken B. Estimation of the domain of attraction for nonlinear autonomous systems using a bezoutian approach// in: Proc. 2016 SICE Int. Symp. on Control Systems. - Nagoya, Japan: Nanzan University, 2016. - P. 1-6.

24. Rezaiee-Pajand M., Moghaddasie B. Estimating the region of attraction via collocation for autonomous nonlinear systems// Struct. Eng. Mech. - 2012. - 41. - P. 263-284.

25. Swiatlak R., Tibken B., Paradowski T., Dehnert R. An interval arithmetic approach for the estimation of the robust domain of attraction for nonlinear autonomous systems with nonlinear uncertainties// in: Proc. 2015 Am. Control Conf., 2015. - P. 2679-2684.

26. Tan W., Packard A. Stability region analysis using polynomial and composite polynomial Lyapunov functions and sum-of-squares programming// IEEE Trans. Automat. Control. - 2008. - 53. - P. 565571.

27. Tорсu U., Packard A.K., Seiler P., Balas G. J. Robust region-of-attraction estimation// IEEE Trans. Automat. Control. - 2010. - 55. - P. 137-142.

28. Wu M., Yang Z., Lin W. Domain-of-attraction estimation for uncertain non-polynomial systems // Commun. Nonlin. Sci. Numer. Simul. - 2014. - 19. - P. 3044-3052.

29. Yang H., Zhang L., Shi P., Hua C. Enlarging the domain of attraction and maximising convergence rate for delta operator systems with actuator saturation// Int. J. Control. - 2015. — 88. — P. 2030-2043.

30. Zecevic A. I., Siljak D. D. Estimating the region of attraction for large-scale systems with uncertainties// Automatica. - 2010. - 46. - P. 445-451.

Yadipour M.

Тебризский университет, Тебриз, Иран

E-mail: m.yadipour92@ms.tabrizu.ac.ir

Hashemzadeh F.

Тебризский университет, Тебриз, Иран

E-mail: hashemzadeh@tabrizu.ac.ir

Baradarannia M.

Тебризский университет, Тебриз, Иран

E-mail: mbaradaran@tabrizu.ac.ir 